\title{
The Trend of Esophageal Cancer Incidence in Iran
}

\author{
Mehdi Jafari ${ }^{1}$, Yousef Moradi ${ }^{1}$, Mahmoud Khodadost ${ }^{1}{ }^{*}$, Eghbal Sekhavati $^{2}$, Hossein Amini Anabad ${ }^{3}$, Kamyar Mansori $^{1}$, \\ Farhad Moradpour ${ }^{1}$, Abdolhalim Rajabi ${ }^{1}$
}

\author{
${ }^{1}$ Department of Epidemiology, Faculty of Health, Iran University of Medical Sciences, Tehran, Iran \\ ${ }^{2}$ Larestan School of Medical Sciences, Larestan, Iran \\ ${ }^{3}$ Health Management Research Center, Baqiyatallah University of Medical Sciences, Tehran, Iran
}

*Corresponding Author: Mahmoud Khodadost, Ph.D. Student, Department of Epidemiology, Faculty of Health, Iran University of Medical Sciences, Tehran, Iran. Email: mahmodkhodadost@yahoo.com

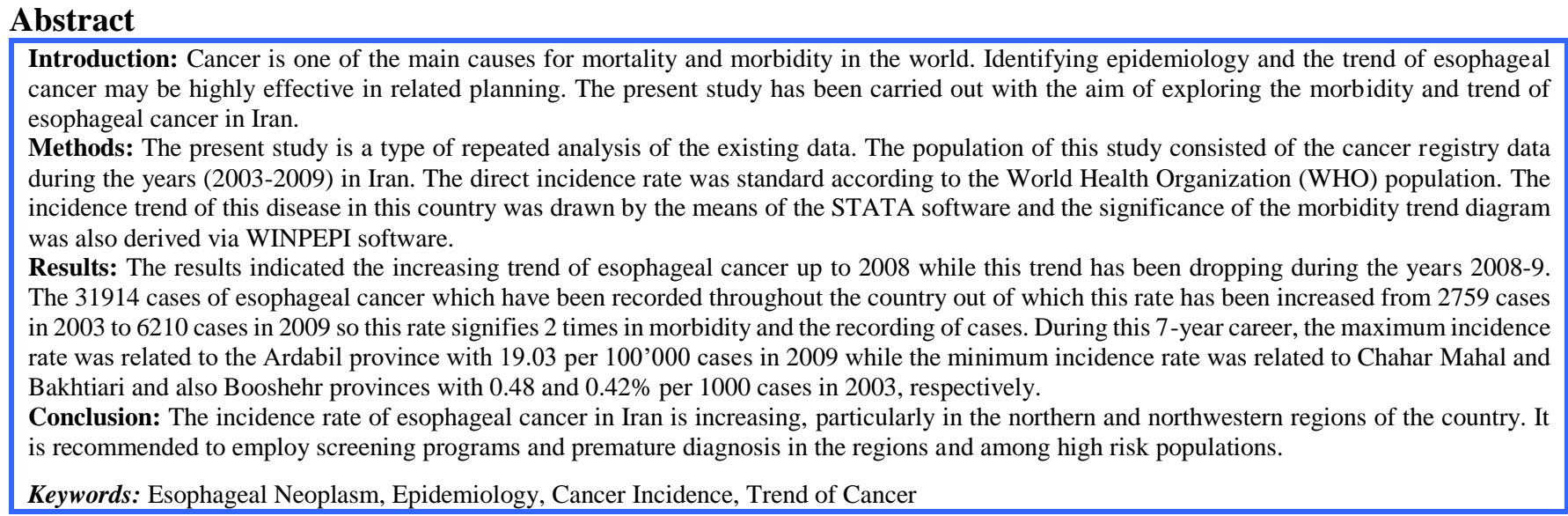

Article History: Received: 7 Feb. 2015; Accepted: 8 May 2015; Online Published: 24 Aug. 2015

Cite this article as: Jafari M, Moradi Y, Khodadost M, Sekhavati E, Amini Anabad H, Mansori K, et al. The Trend of Esophageal Cancer Incidence in Iran. Int J Travel Med Glob Health. 2015;3(3):127-31.

\section{Introduction}

Cancer is considered to be one of the main causes of mortality throughout the world. After cardiovascular diseases, it is the second most frequent morbidity factor in developed countries and the third greatest mortality factor in the less-developed nations [1]. According to a report from the WHO organization in 2012, 2.8million cases of mortality (about $13 \%$ of total mortalities) have been recorded to be from cancer while about $70 \%$ of total mortalities by cancers occur in the counties with low and middle income [2, 3]. Similarly, it has been estimated that the total cancer mortality rates will reach more than 13.1 million cases by 2030 [4]. According to the latest statistics from the National Cancer Registration Center in 2008, 74067 new cases of cancer have been recorded out of which 41169 cases $(55 \%)$ were males and 32898 cases $(45 \%)$ were females. The registration of 90 thousands of new cases of cancer in the country per year and mortality rate of 55 cases of cancer signifies the fact that the morbidity of cancer in Iran is an alarming issue. More than $30 \%$ of cancers may be prevented with healthy life cycles and/ or immunization against infections of cancer factors (Human Papilloma Virus HPV and Hepatitis B- Virus HBV). Also, $30 \%$ of these cases can be cured in the case of premature diagnosis [5]. Among cancers, esophageal cancer is crucially important. This cancer is the ninth most fatal cancer in the world and is ranked at fifth position in the developed and developing countries [6]. This disease is extremely lethal and there is obvious differences among the rate of its mortality in various limited geographical zones. Such a difference may be due to the ecologic factors and/ or genetic susceptibility. The regions with high rate of mortality from esophageal cancer are situated in Central Asia (Asian Belt), France, Italy, Brazil, Eastern and Southern Africa. The noticeable point is that in these countries we witness the presence of focal points with very high risks of morbidity along with less morbid zones [7-9]. The studies show that during recent decades esophageal cancer has decreased in the Asian Belt Zone and the occurrence of this cancer has reduced to about $50 \%$ in china. This reduction in the morbidity of cancer has been also seen in Africa and Europe (except in adult males). In contrast, the sudden rise in the occurrence of the disease was seen among the black race in USA. Such time developments are totally considered as the evidences for change in the related exposures to the given disease $[6,8-10]$. The maximum reported rate of morbidity from esophageal cancer in the world belongs to a report from the Cancer Center in the Caspian Sea. In this report, the rate of the occurrence of esophageal cancer has been reported to be 165 in age group (35 years and older) for males and 195 for females as per 100'000 cases in Gonbad city, Iran [11]. Based on the report of Globocan 2008, the Age Standardized Incidence Rate (ASR) in developed countries has been reported to be 1.2 in females and 6.5 among males and this rate is 10.1 for males and 4.2 for females in the world. According to the latest statistics from the National Cancer Registration Center, with Age Standardized Rate (ASR) of 5.88 among females this rate is ranked in the fifth position while ASR of 6.15 among males is placed at the eighth position [5, 11]. Esophageal cancer has remarkable discrepancies in Iran in such a way that after gastric and skin cancers in the Ardabil province, this cancer is ranked as the second most popular cancer after gastric cancer among 
females [12].Most northern and northwestern areas in Iran are at high risk of esophagus and gastric cancer, while the central and western provinces are at medium risk and the southern regions are at a low risk [13]. Age and gender are some of the individual characteristics which may influence the occurrence of this cancer. The rate of morbidity from esophageal cancer rises as age increases. In the sixth decade of lifetime, this rate is considerably higher. The reported cases of the cancer in the Cancer Registration Center in North of Iran were older than 40 [14]. There are different statistics regarding gender ratio (male to female) in morbidity from esophageal cancer. While, this ratio is greater than 10 in France, this rate has been reported even less than 1 in the North of Iran [14, 15]. Smoking, tobacco use and alcohol abuse are assumed to be other risk factors in the occurrence of this cancer. In all forms, they are accompanied with high risks of morbidity from esophageal cancer. Alcohol abuse is not considered as the health problem in high risk zones of Central Asia. However, it seems that opium abuse (in different forms) is assumed to be one of the main risk factors in the occurrence of esophageal cancer. The opium residue is widely used in these regions, especially in the North of Iran. According to in vitro studies, it has a very strong carcinogenic effect [16]. Likewise, several studies in Iran, USA, and Singapore have provided reliable evidences as per the high risks of morbidity of this cancer among lower social classes [17]. Malnutrition, special nutrition habits, alcohol abuse and opium use may increase the high risks of occurrence of esophageal cancer in the aforementioned groups. Poor literacy and unfavorable socioeconomic status are associated with the occurrence of esophageal cancer in the North of Iran. Therefore, they are attributed to scarcity of some important vitamins such as vitamins A and B2 [17-19]. Due to the existing reasons, this type of cancer is probably frequent in the country, but there has been no study which deals with the trend of this serious disease and the variance of its occurrence in the recent decades as well as its epidemiology in the country. Nevertheless, few studies have been conducted regionally with small quantity of cases. Alternately, the presence of epidemiologic information requires successful planning. Therefore, this study has been carried out in order to explore the epidemiology of esophageal cancer and determine the trend changes of the disease in Iran in the years 2003-2009.

\section{Methods}

The present study is a cross-sectional research conducted by the analysis of the existing data. In this study, the data were obtained from the National Cancer Registration System about cancer cases as well as information from diseases administration center in the Ministry of Health and Medical
Sciences. After receiving data from the national pathology centers, disease administration center reviews them in terms of proper encoding and revises the existing the duplicated cases and defects in data by means of Pars Software. In this research, all of national registered cases have been examined during the years 2003-2009 across the country as well as the isolated statistics regarding esophageal cancer for each province. The reported incidence rates have been standardized by means of direct method and based on standard population of the WHO. The extracted data of the whole country have been explored and studied according to the number of cases and standardized incidence rates of the provinces. After data collection, disease trend during the years of conducting the study and determining the frequency distribution of esophageal cancer were drawn based on different zones. Also, the assessment of significance of changes in trend of incidence cases diagrams was evaluated by means of WINPEPI software.

\section{Results}

Study on the statistics of the Cancer Registration Center indicates that during the years 2003-2009, the age standardized incidence rate (ASR) of esophageal cancer had an incremental trend among females and males in the country until 2008 while this trend has declined since 2008 (Diagram 1).

Based on these statistics during this period in Iran, 21483 cases of esophageal cancer have been recorded in the country. According to their classification in terms of gender, 9760 cases were males and 11723 were females. The maximum rate of new cases of this cancer was reported to be 3931 cases in 2008 and the minimum rate was 2339 cases in 2003 (Table 1).

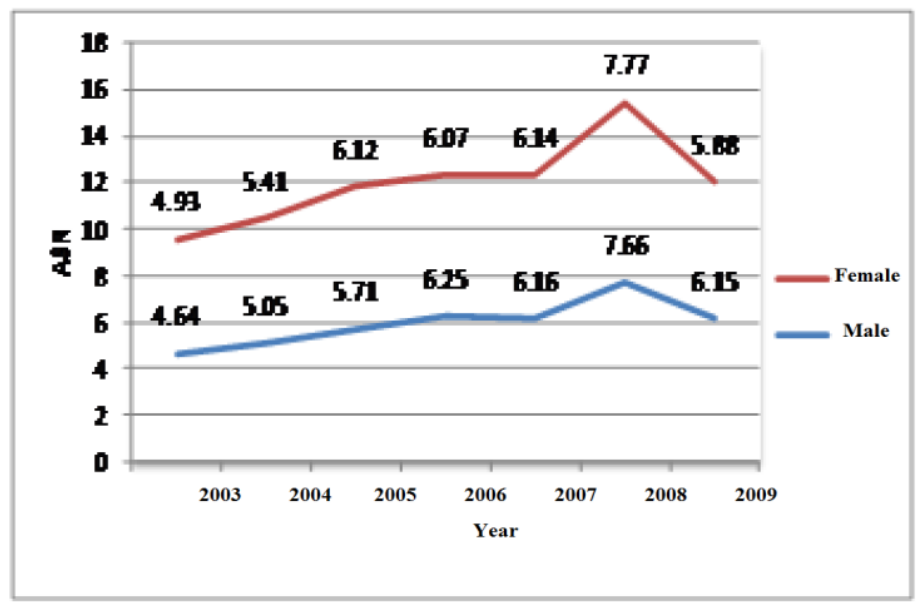

Diagram 1. Trend of variance in morbidity rate of esophageal cancer in Iran during years 2003-2009

Table 1. Occurrence of esophageal cancer in Iran based on statistics from Cancer Registration Center

\begin{tabular}{|c|c|c|c|c|}
\hline \multirow{2}{*}{ Statistics of Cancer Registration Center } & \multicolumn{2}{|c|}{ Age Standardized Rate (ASR) } & \multicolumn{2}{|c|}{ Quantity (Percent) } \\
\hline & Female & Male & Female & Male \\
\hline 2003 & 4.93 & 3.65 & $1093(6.49 \%)$ & $1246(5.76 \%)$ \\
\hline 2004 & 4.41 & 5.05 & $1192(5.82 \%)$ & $1392(5.21 \%)$ \\
\hline 2005 & 6.12 & 5.71 & $1429(5.83 \%)$ & $1617(5.16 \%)$ \\
\hline 2006 & 6.07 & 6.25 & $1403(5.39 \%)$ & $1772(5.25 \%)$ \\
\hline 2007 & 6.14 & 6.16 & $1412(5.155)$ & $1699(4.91 \%)$ \\
\hline 2008 & 7.77 & 7.66 & $1782(5.26 \%)$ & $2149(5.06 \%)$ \\
\hline 2009 & 5.88 & 6.15 & $1449(4.4 \%)$ & $1848(4.5 \%)$ \\
\hline Total & & & 9760 & 11723 \\
\hline
\end{tabular}


Based on the statistics from the Cancer Registration Center, the highest age standardize incidence rate (ASR) was $7.77 \%$ per 100,000 populations among females during this period while the lowest rate of ASR was 4.93 that has been observed in 2003. Similarly, study on reports from the Cancer Registration Center in the provinces of the countries during years 2003-9 has shown that the ASR of esophageal cancer in 30 provinces in Iran (Table 2) in which the highest ASR was related to the Ardabil province with 19.03 per 100000 populations in 2008 (for females) and the lowest ASR in ChaharMahal and Bakhtiari and Booshehr provinces with 0.48 and 0.42 per 100000 populations.

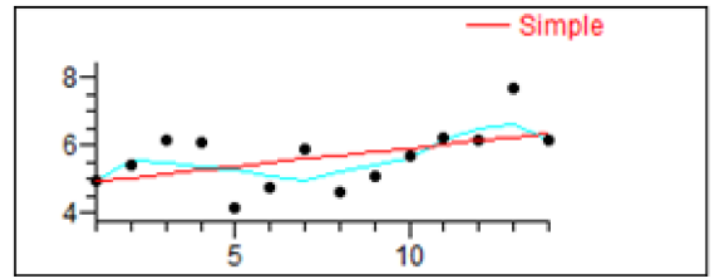

Chi-Sq $=48.17$ (DF: 1); $\mathrm{P}=0.000$ (3.9E-12)

Diagram 2. Chart of significance of variance trend for occurrence of esophageal cancer in Iran

This statistics show that the northern and northwestern provinces of the country have the highest incidence rate of esophageal cancer and the trend of cancer occurrence in these provinces has an uphill trend compared to the other zones.

The statistical significance of changes in trend diagram of Age Standardized Incidence Rate (ASR) of esophageal cancer per 100 thousands populations was assessed by means of WINPEPI software during this time interval where their results are given in Diagram 2 separately based on gender.
According to this diagram, changes in the trend of esophageal cancer occurrence in both genders is remarkably significant $(\mathrm{P}<0.0001)$.

\section{Discussion}

Based on the data collected from Cancer Registration Center, the age standardized incidence rate (ASR) of esophageal cancer in Northern provinces is as follows: Golestan (males: 12.30 and females: 9.91), Mazandaran (males: 9.44 and females: 7.58), and Ardebil provinces (males: 8.15 and females: 9.91) where this finding is aligned with the acquired results from other studies such as Zendehdel, Semnani, and Malekzadeh in Golestan, Hajian in Mazandaran, Sokooti in Ardebil, and KhabazKhoob in Tehran $[5,12,17]$.

Regarding the epidemiology of esophageal cancer, the north of Iran is not only one of the zones, which are situated on esophageal cancer belt, but also is assumed as one of the focal points for esophageal cancer in the world. In Turkmen Sahara Region this rate is at the peak. According to the studies conducted on these data and also various epidemiologic studies on littoral regions of Caspian Sea, there is stark difference in rate of esophageal cancer occurrence in the limited geographical zones and such a difference may be attributed to ecologic factors and/ or genetic susceptibilities.

It is noteworthy that the esophageal cancer is one of the cancers in which the ecologic factors intervene in its occurrence in addition to genetic effect and these results have been confirmed by the Besharat in Golestan province [12, 13, 17, 18]. According to these statistics, the gender ratio of esophageal cancer was different in various provinces but this rate was often higher among males than in females and this

Table 2. Standardized Mortality Rate of esophageal cancer in national provinces during years 2003-2009

\begin{tabular}{|c|c|c|c|c|c|c|c|c|c|c|c|c|c|c|}
\hline \multirow{2}{*}{ Province } & \multicolumn{2}{|c|}{2003} & \multicolumn{2}{|c|}{2004} & \multicolumn{2}{|c|}{2005} & \multicolumn{2}{|c|}{2006} & \multicolumn{2}{|c|}{2007} & \multicolumn{2}{|c|}{2008} & \multicolumn{2}{|c|}{2009} \\
\hline & $\mathbf{F}$ & $\mathbf{M}$ & $\mathbf{F}$ & $\mathbf{M}$ & $\mathbf{F}$ & $\mathbf{M}$ & $\mathbf{F}$ & $\mathbf{M}$ & $\mathbf{F}$ & M & $\mathbf{F}$ & M & $\mathbf{F}$ & M \\
\hline Eastern Azerbaijan & 1.49 & 1.99 & 7.99 & 8.39 & 3.13 & 2.17 & 5.30 & 4.53 & 9.92 & 10.76 & 13.52 & 12.07 & 11.57 & 12.09 \\
\hline Western Azerbaijan & 9.70 & 10.27 & 9.04 & 9.77 & 11.27 & 8.70 & 9.95 & 9.70 & 12.04 & 9.68 & 12.22 & 8.20 & 19.32 & 11.52 \\
\hline Ardebil & 8.14 & 8.15 & 16.13 & 4.18 & 13.76 & 11.64 & 6.07 & 6.25 & 12.83 & 10.23 & 19.03 & 14.60 & 9.91 & 8.15 \\
\hline Isfahan & 1.58 & 1.76 & 1.92 & 3.06 & 1.92 & 2.74 & 2.21 & 3.21 & 2.17 & 3.20 & 1.83 & 3.83 & 2.53 & 2.83 \\
\hline Ilam & 9.44 & 4.15 & 9.18 & 4.62 & 9.84 & 6.81 & 2.64 & 5.52 & 8.26 & 10.28 & 10.74 & 11.83 & 6.84 & 4.52 \\
\hline Booshehr & 0.56 & 1.49 & 0.68 & 1.60 & 0.66 & 0 & 0.56 & 3.69 & 1.28 & 1.12 & 0.72 & 3.45 & 1.13 & 0.42 \\
\hline Tehran & 5.61 & 4.93 & 2.18 & 2.27 & 3.31 & 3.26 & 2.49 & 3.75 & 2.64 & 2.64 & 5.29 & 5.16 & 4.08 & 4.18 \\
\hline ChaharMahal\&Bakhtiari & 0 & 0 & 0.48 & 1.64 & 1.65 & 1.96 & 1.28 & 3.61 & 0.77 & 2.77 & 1.06 & 4.23 & 0.78 & 3.00 \\
\hline Southern Khorasan & & & & & 10.49 & 4.88 & 4.35 & 4.01 & 0.41 & 1.10 & 11.39 & 10.95 & 10.62 & 7.82 \\
\hline RazaviKhorasan & 13.67 & 11.04 & 12.74 & 9.86 & 8.90 & 10.33 & 13.87 & 13.59 & 13.79 & 11.28 & 14.32 & 13.93 & 11.49 & 12.95 \\
\hline Northern Khorasan & & & & & 12.62 & 10.59 & 9.15 & 7.65 & 6.35 & 12.77 & 14.42 & 11.42 & 8.35 & 14.19 \\
\hline Khuzestan & 1.48 & 2.14 & 2.04 & 1.89 & 2.27 & 1.82 & 1.76 & 1.58 & 1.97 & 2.49 & 3.53 & 3.59 & 1.88 & 2.75 \\
\hline Zanjan & 5.33 & 6.41 & 8.65 & 5.42 & 3.44 & 6.24 & 7.57 & 6.27 & 7.48 & 6.76 & 10.19 & 6.74 & 5.14 & 7.92 \\
\hline Semnan & 6.48 & 5.22 & 5.93 & 6.64 & 14.92 & 5.02 & 8.72 & 7.94 & 6.97 & 8.85 & 12.10 & 10.53 & 5.52 & 4.74 \\
\hline Sistan\& Baluchistan & 2.11 & 1.84 & 2.08 & 1.21 & 3.19 & 2.50 & 3.47 & 2.23 & 2.73 & 2.58 & 4.45 & 2.74 & 4.01 & 2.82 \\
\hline Fars & 1.34 & 2.53 & 1.32 & 2.08 & 1.20 & 2.28 & 1.46 & 2.05 & 2.00 & 4.09 & 2.91 & 3.68 & 2.29 & 2.89 \\
\hline Qazvin & 5.74 & 6.16 & 4.36 & 3.83 & 4.35 & 3.81 & 6.46 & 5.29 & 3.21 & 3.80 & 5.63 & 8.08 & 4.62 & 6.21 \\
\hline Qom & 4.42 & 5.35 & 4.94 & 5.73 & 5.13 & 5.65 & 4.29 & 6.04 & 6.28 & 5.16 & 4.49 & 5.02 & 4.39 & 3.05 \\
\hline Golestan & 5.56 & 5.01 & 7.62 & 8.33 & 11.04 & 11.74 & 11.79 & 13.99 & 11.04 & 13.73 & 12.19 & 14.80 & 10.27 & 12.30 \\
\hline Guilan & 3.03 & 4.41 & 4.03 & 4.86 & 6.13 & 7.42 & 5.79 & 6.85 & 7.14 & 8.87 & 5.99 & 9.31 & 4.82 & 7.76 \\
\hline Lorestan & 3.93 & 5.02 & 7.66 & 6.57 & 4.30 & 4.02 & 5.98 & 6.89 & 7.58 & 7.13 & 11.08 & 11.83 & 7.16 & 8.80 \\
\hline Mazandaran & 7.28 & 6.26 & 7.27 & 7.17 & 7.51 & 9.58 & 8.68 & 10.16 & 8.78 & 7.28 & 10.68 & 10.93 & 7.85 & 9.44 \\
\hline Markazi & 0.92 & 1.80 & 2.23 & 2.29 & 1.95 & 2.19 & 1.97 & 3.89 & 4.16 & 3.53 & 2.06 & 2.16 & 3.93 & 2.95 \\
\hline Hormozgan & 0.52 & 0 & 2.14 & 1.30 & 1.26 & 0.95 & 2.37 & 1.42 & 2.59 & 1.33 & 2.22 & 0.99 & 1.93 & 1.39 \\
\hline Hamedan & 3.76 & 2.77 & 2.82 & 2.44 & 4.89 & 3.19 & 4.48 & 4.04 & 4.39 & 6.35 & 4.06 & 5.63 & 5.43 & 5.69 \\
\hline Kurdistan & 13.70 & 7.48 & 16.09 & 7.76 & 11.02 & 8.74 & 13.06 & 11.18 & 12.09 & 9.94 & 15.97 & 11.95 & 14.73 & 11.77 \\
\hline Kerman & 1.88 & 1.05 & 1.84 & 0.97 & 2.15 & 1.81 & 2.22 & 1.59 & 1.78 & 1.80 & 2.38 & 2.58 & 2.83 & 2.41 \\
\hline Kermanshah & 7.10 & 5.65 & 5.17 & 6.48 & 7.15 & 5.29 & 5.26 & 7.31 & 7.33 & 5.37 & 7.61 & 6.53 & 7.05 & 4.89 \\
\hline Kokiluyeh\& Boyer Ahmad & 0 & 0 & 0.53 & 4.49 & 2.39 & 0.48 & 1.55 & 2.34 & 2.29 & 3.90 & 1.92 & 2.60 & 2.41 & 5.14 \\
\hline Yazd & 3.54 & 1.60 & 2.80 & 1.19 & 0.81 & 2.32 & 1.62 & 2.81 & 4.72 & 4.96 & 4.83 & 2.27 & 4.86 & 2.72 \\
\hline
\end{tabular}


indicates that males are more susceptible to this cancer than females. This finding is consistent with the studies conducted by Esmailnasab and Sajadi in Iran and William $\mathbf{J}$ and Coleman MP in USA $[9,11,20]$.

Examining the diagram of total ASR of cancers in this country indicates that as age increases, the rate of occurrence of this cancer rises and the maximum age standardized incidence rate of esophageal cancer occurs in women and men at the age of 80 and then it is reduced. This rate is decreased because of lack of reference of patients to diagnostic centers, inaccessibility of old persons to diagnostic facilities or lack of execution of diagnostic experiments by physicians at such ages. This finding corresponds to the results of the studies of Esmailnasab in Sanandaj and Khabaz in Tehran [12]. According to the published reports by the National Cancer Registration Center, esophageal cancer includes $4.4 \%$ of total womenrelated cancers and it has been known as the fifth most prevalent cancer among Iranian females. Similarly, with the rate of $5.4 \%$ of total men- related cancers, this cancer is the eighth most prevalent cancer among Iranian males. Based on these reports, rate of morbidity and occurrence of esophageal cancer varies according to geographical zone, race and life conditions, socio- economic status, and lifestyle and these findings have been verified in the study by Gholipoor from Tabriz, Malekshah et al in Golestan, and Hemati in Ilam [14, $15,21]$.

In the study by Anita AM Botterweck in Europe carried out in 10 European countries, it has been concluded that the mortality rate of this cancer has had a growing trend in the northern and southern and western European countries including Denmark, Belgium, Germany, and UK. Except in some central regions like Switzerland and Basel city in this country where the cancer has had been relatively fixed and even with a decremented trend, this finding is consistent with the studies conducted in other countries. For example, it is in line with the studies conducted by Susan S and Katrina F in USA, Shuzheng Liu in China and Hong Kong, and A. Paloma Vizcaino in Africa [22, 23].

In the study by Anita AM Botterweck in Europe carried out in 10 European countries, it has been concluded that the mortality rate of this cancer has had a growing trend in northern and southern and western European countries including Denmark, Belgium, Germany, and UK. Except in some central regions like Switzerland and Basel city in this country where the cancer has had been relatively fixed and even with a decremented trend, this finding is consistent with the studies conducted in other countries. For example, it is in line with the studies conducted by Susan S and Katrina F in USA, Shuzheng Liu in China and Hong Kong, and A. Paloma Vizcaino in Africa [22, 23].

Considering the statistics from the Cancer Registration Center, this cancer has had an ascending trend in Iran. These findings are consistent with the statistics presented in various points of the world signifying a growing trend in such a way that according to the statistics from WHO Organization, the occurrence of this cancer has an up-surging trend in the recent decades [25]. Based on the studies conducted by Chiang et al. in Hong Kong, the morbidity rate of this cancer in Hong Kong during the recent four decades has had no identical trend. However, during the recent years this cancer has had a relatively fixed trend and has reduced in some cases. These findings are in line with the studies conducted in ten European countries and the results may be used for improving medical healthcare conditions and for changing lifestyles [26]. In a national study that was executed by Zendehdel in Iran, the occurrence rate of this cancer in Iran has increased both in males and females from 2004 to 2006. However, no upward trend has been observed for 30 provinces. Similar results were derived from studies done by Hajian in Mazandaran, Semnani in Golestan, Tayebi in Babolsar, and Esmailnasab in Kurdistan. All these researches have signified an incremental trend of this cancer in the country and this complies with the official published reports by the National Cancer Registration Center [11, 27]. In a study that was conducted by Eslami et al. in Iran, the relation between elements among social capitals with the occurrence and risk factors of esophageal cancer have been examined. They have concluded that the elements of social capitals including training social welfare, marriage, and socioeconomic status etc. has had a reverse and significant relation with the occurrence of this cancer $[26,28]$.

\section{Conclusion}

With respect to the statistics from the Cancer Registration Center in Iran, like all the other regions in the world, the trend of this disease is growing. It has been proved that the occurrence of this cancer is higher in the northern strip compared to the other regions of the country. At the same time, the burden caused by the occurrence of this disease and the etiologic investigations in the country particularly in the central regions seems necessary. Thus, it is suggested that etiologic and pathologic studies should be designed and conducted with ongoing plans to raise the awareness of people in these regions concerning the significant relations between this cancer, unhealthy lifestyle and lifestyle. In addition, improving eating habits and enhancing health and leading a more active life will reduce the occurrence of this cancer in the country, especially in the regions susceptible to high risks.

\section{Acknowledgments}

The authors express their gratitude to all of the colleagues in this study and all the staff at the Cancer Office in the Ministry of Health of Iran.

\section{Authors' Contributions}

All authors have equally contributed to this study.

\section{Financial Disclosure}

All authors declare that they have no conflict of interest.

\section{Funding/Support}

This article has been extracted from research project approved and supported by the Iran University of Medical Sciences.

\section{References}

1. Bhurgri Y, Bhurgri A, Hasan SH. Comparability and Quality Control in Cancer Registration; Karachi (data monitoring 1995-2001). J Pak Med Assoc. 2002;52(7):301-7.

2. Gallo A, Cha C. Updates on esophageal and gastric cancers. World $\mathbf{J}$ Gastroenterol. 2006;12(20):3237-42.

3. Valsecchi MG, Steliarova-Foucher E. Cancer registration in developing countries: luxury or necessity?. Lancet Oncol. 2008;9(2):159-67.

4. Siesling S, Louwman WJ, Kwast A, van den Hurk C, O'Callaghan M, Rosso S, et al. Uses of cancer registries for public health and clinical research in Europe: Results of the European Network of Cancer Registries survey among 161 population-based cancer registries 
during 2010-2012. Eur J Cancer. 2015;51(9):1039-49.

5. Yahyapour Y, Shamsi-Shahrabadi M, Mahmoudi M, Motevallian A, Siadati S, Shefaii S, et al. High-risk and low-risk human papillomavirus in esophageal squamous cell carcinoma at Mazandaran, Northern Iran. Pathol Oncol Res. 2013;19(3):385-91.

6. Islami F, Pourshams A, Nasrollahzadeh D, Kamangar F, Fahimi S, Shakeri R, et al. Tea drinking habits and oesophageal cancer in a high risk area in northern Iran: population based case-control study. BMJ. 2009;338:b929.

7. Islami F, Malekshah AF, Kimiagar M, Pourshams A, Wakefield J, Goglani G, et al. Patterns of food and nutrient consumption in northern Iran, a high-risk area for esophageal cancer. Nutr Cancer. 2009;61(4):475-83.

8. Abedi-Ardekani B, Kamangar F, Hewitt SM, Hainaut P, Sotoudeh M, Abnet CC, et al. Polycyclic aromatic hydrocarbon exposure in oesophageal tissue and risk of oesophageal squamous cell carcinoma in north-eastern Iran. Gut. 2010;59(9):1178-83.

9. Malekshah AF, Kimiagar M, Pourshams A, Yazdani J, Kaiedi Majd S, Goglani G, et al. Vitamin deficiency in Golestan Province, northern Iran: a high-risk area for esophageal cancer. Arch Iran Med. 2010;13(5):391-4.

10. Taghavi N, Biramijamal F, Sotoudeh M, Moaven O, Khademi H, Abbaszadegan MR, et al. Association of p53/p21 expression with cigarette smoking and prognosis in esophageal squamous cell carcinoma patients. World J Gastroenterol. 2010;16(39):4958-67.

11. Hajizadeh B, Jessri M, Moasheri SM, Rad AH, Rashidkhani B. Fruits and vegetables consumption and esophageal squamous cell carcinoma: a case-control study. Nutr Cancer. 2011;63(5):707-13.

12. Roshandel G, Majdzadeh R, Keshtkar A, Aramesh K, Sedaghat SM, Semnani S. Healthcare utilization in patients with esophageal cancer in a high risk area in northeast of Iran. Asian Pac J Cancer Prev. 2011;12(9):2437-42.

13. Khodadost M, Yavari P, Babaei M, Mosavi-Jarrahi A, Sarvi F, Mansori K, et al. Estimating the completeness of gastric cancer registration in Ardabil/Iran by a capture-recapture method using population-based cancer registry data. Asian Pac J Cancer Prev. 2015;16(5):1981-6.

14. Etemadi A, Abnet CC, Golozar A, Malekzadeh R, Dawsey SM. Modeling the risk of esophageal squamous cell carcinoma and squamous dysplasia in a high risk area in Iran. Arch Iran Med. 2012;15(1):18-21.

15. Keshavarzi B, Moore F, Najmeddin A, Rahmani F. The role of selenium and selected trace elements in the etiology of esophageal cancer in high risk Golestan province of Iran. Sci Total Environ. 2012;433:89-97.
16. Ghasemi-Kebria F, Joshaghani H, Taheri NS, Semnani S, Aarabi M, Salamat F, et al. Aflatoxin contamination of wheat flour and the risk of esophageal cancer in a high risk area in Iran. Cancer Epidemiol. 2013;37(3):290-3.

17. Ghasemi-Kebria F, Roshandel G, Semnani S, Shakeri R, Khoshnia M, Naeimi-Tabiei M, et al. Marked increase in the incidence rate of esophageal adenocarcinoma in a high-risk area for esophageal cancer. Arch Iran Med. 2013;16(6):320-3.

18. Roshandel G, Khoshnia M, Sotoudeh M, Merat S, Etemadi A, Nickmanesh A, et al. Endoscopic screening for precancerous lesions of the esophagus in a high risk area in Northern Iran. Arch Iran Med. 2014;17(4):246-52.

19. Razavi SM, Khodadost M, Sohrabi M, Keshavarzi A, Zamani F, Rakhshani N, et al. Accuracy of endoscopic ultrasonography for determination of tumor invasion depth in gastric cancer. Asian Pac J Cancer Prev. 2015;16(8):3141-5.

20. Trivers KF, Sabatino SA, Stewart SL. Trends in esophageal cancer incidence by histology, United States, 1998-2003. Int J Cancer. 2008;123(6):1422-8.

21. Eslamian G, Jessri M, Hajizadeh B, Ibiebele TI, Rashidkhani B. Higher glycemic index and glycemic load diet is associated with increased risk of esophageal squamous cell carcinoma: a case-control study. Nutr Res. 2013;33(9):719-25.

22. Haghdoost AA, Hosseini H, Chamani G, Zarei MR, Rad M, Hashemipoor M, et al. Rising incidence of adenocarcinoma of the esophagus in Kerman, Iran. Arch Iran Med. 2008;11(4):364-70.

23. Liu S, Dai JY, Yao L, Li X, Reid B, Self S, et al. Esophageal Adenocarcinoma and Its Rare Association with Barrett's Esophagus in Henan, China. PLoS One. 2014;9(10):e110348.

24. Ke L. Mortality and incidence trends from esophagus cancer in selected geographic areas of China circa 1970-90. Int J Cancer. 2002;102(3):271-4.

25. Parkin DM, Bray F, Ferlay J, Pisani P. Global cancer statistics, 2002. CA Cancer J Clin. 2005;55(2):74-108.

26. Jansson C, Johansson AL, Nyrén O, Lagergren J. Socioeconomic factors and risk of esophageal adenocarcinoma: a nationwide Swedish case-control study. Cancer Epidemiol Biomarkers Prev. 2005;14(7): 1754-61.

27. Hakami R, Mohtadinia J, Etemadi A, Kamangar F, Nemati M, Pourshams A, et al. Dietary intake of benzo(a)pyrene and risk of esophageal cancer in north of Iran. Nutr Cancer. 2008;60(2):216-21.

28. Islami F, Kamangar F, Nasrollahzadeh D, Aghcheli K, Sotoudeh M, Abedi-Ardekani B, et al. Socio-economic status and oesophageal cancer: results from a population-based case-control study in a highrisk area. Int J Epidemiol. 2009;38(4):978-88. 